\title{
Recurrent Empyema in a Healthy 7 Year Old Boy
}

\author{
Gihad Alsaeed*1, Mohammed Mahmoud Elbarrawy ${ }^{2}$, Mohamed \\ Alsaeed $^{3}$, IbrahimAlsaeed ${ }^{4}$ \\ ${ }^{1}$ Doctor Sulaiman Alhabib Hospital -TKH, Pediatric Department, Riyadh, Saudia \\ Arabia \\ ${ }^{2}$ Faculty of Medicine, Cairo University, Cairo, Egypt \\ ${ }^{3}$ Faculty of Medicine, University of Pavia, Pavia, Italy \\ ${ }^{4}$ Faculty of Medicine, University of Milano, Milano Italy \\ *Corresponding Author: Gihad Alsaeed, Doctor Sulaiman Alhabib Hospital \\ -TKH, Pediatric Department, Riyadh, Saudia Arabia.
}

Received: May 03, 2020

Published: June 23, 2020

(C) All rights are reserved by Gihad Alsaeed., et al.

\section{Abstract}

Parapneumonic effusion and subsequent empyema are known complications of bacterial pneumonia. It is seen in approximately 2 to 12 percent of children with pneumonia and up to 28 percent of those requiring hospitalization as pneumonia [1,2]. Recurrent empyema is recurrence of empyema after its apparent healing. The incidence of recurrent empyema in children is $8.9 \%$ of empyema cases. Possible causes of recurrent empyema are recurrent pneumonia, chest surgery, immunodeficiency and chronic lung diseases. Surgical treatment of empyema is preferred by many experts more than conservative treatment alone. Recurrent empyema often needs surgical interventions to treat the cause and complications to achieve complete recovery [1,2]. We present a case of recurrent empyema in a previously healthy 7-year-old boy who had no underlying etiology inspite of extensive investigation and who recovered completely without open thoracotomy or video-assisted thoracoscopic surgery (VATS).

Keywords: Recurrent Empyema; Children; Pleural Effusion; Bacterial Pneumonia

\section{Introduction}

Pleural effusion is an excess of fluid that accumulates in the pleural cavity. This excessive fluid may impair breathing by limiting the expansion of the lungs [2]. Pleural effusion can be classified into many types according to the nature of the fluid and what caused its entry into the pleural space: Hydrothorax (serous fluid), Hemothorax (blood), Chylothorax (chyle) or Empyema (pus) [3]. Empyema is the presence of bacterial organisms on Gram stain and/or grossly purulent fluid in the pleural cavity. Complicated pneumonia refers to pneumonia with any complication, including loculated para pneumonic effusion; empyema or more rarely, pneumothorax, necrotizing pneumonia, or lung abscess. The most common precursor of empyema thoracic is bacterial pneumonia and subsequent para-pneumonic effusion. Other rare causes of empyema include bronchogenic carcinoma, esophageal rupture, blunt or penetrating chest trauma, mediastinitis with pleural extension, infected congenital cysts of the airway and esophagus, extension from sources below the diaphragm, cervical and thoracic spine infections, as well as postsurgical etiologies [5]. Common causative organisms of empyema are Streptococcus pneumonia and Staphylococcus aureus, Escherichia coli, Haemophilus influenza and Klebsiella pneumonia, Streptococcus pyogenes and uncommon causative organisms such as Mycobacterium tuberculosis and Cryptococcus neoformans [5]. Community-associated MRSA is a common cause of parapneumonic effusion/empyema. S. pneumoniae (Pneumococcus) has been the most common pathogen causing para pneumonic effusions and empyema, but its prevalence is decreasing with changes in pneumococcal vaccines [8]. Common serotypes that cause invasive pneumococcal disease are $1,3,7 \mathrm{~F}$ or $19 \mathrm{~A}[1,9]$. These serotypes were not included in the 7 -valent pneumococcal 
conjugate vaccine (PCV7) but are in the PCV13 conjugated vaccine [7]. The American Thoracic Society has described three stages of empyema, exudative, fibrinopurulent and organized stage. Exudative fluid is low in cellular content with normal chemistry and negative gram stain. This stage tends to last approximately 24 to 72 hours [9]. The second stage is known as the fibrinopurulent stage, which is triggered by bacterial invasion of the pleural space, causing empyema. In fibrinopurulent stage leukocytes and LDH accumulate in pleural fluid, then pus and fibrin start to cover the pleura with the formation of loculations. This stage may last 7 to 10 days [9]. The third organizational stage is characterized by thick exudate and heavy sediment like a thick peel formed by fibroblasts [4]. This stage typically occurs by two to four weeks after initial development of the empyema [4].

The clinical presentation in children with empyema is most often similar to that of pneumonia. These include high grade fever with chills, rigors, cough, tachypnea, breathlessness and chest pain. Chest examination typically reveals decreased unilateral chest expansion with reduced breath sounds on auscultation. Tactile fremitus is increased in pneumonia and following the development of pleural fluid, these decrease with an associated stony dull percussion. Typically, a persistent fever despite 48 hours of appropriate antibiotic treatment, together with a change in physical signs should alert the clinician to the possible development of pleural fluid as a complication of pneumonia [8]. Empyema may cause complications like Bronchopleural fistula, pyopneumothorax, purulent pericarditis, pulmonary abscess and osteomyelitis of ribs [3].

Chest X-ray should be performed in all children in whom an empyema is suspected [8]. Pleural ultrasound imaging (US) has become the cornerstone for evaluation of pleural effusions. Contrastenhanced CT with tissue phase is a valuable imaging tool in the assessment of empyema. While it is routine to perform a diagnostic pleural tap and utilize biochemical markers to guide management in adults, it is not recommended in children as it requires cooperation or sedation. Therefore, pleural fluid investigations in childhood should only be undertaken if the empyema requires drainage as part of therapy [8]. The decision to intervene with drainage is easy in a child with moderate to severe respiratory distress, a large pleural effusion and ongoing sepsis. The decision is much more difficult in those early on in the disease with mild respiratory distress and a small effusion because some parapneumonic effusions and early empyemas may resolve spontaneously and it is impossible to predict which child will progress to a more complicated empyema [8]. The presence of pus, positive Gram's stain, or culture in the pleural fluid analysis establishes the diagnosis of empyema and should prompt tube thoracostomy drainage [5]. In the absence of these factors, a large meta-analysis suggests that pleural $\mathrm{PH}<7.2$ is the next most useful predictor of a complicated clinical course in the absence of adequate chest tube drainage [9]. If pleural PH is not measured, a pleural fluid glucose value of $<40 \mathrm{mg} / \mathrm{dl}$ should prompt chest tube drainage [10]. Likewise, a pleural fluid LDH > $1000 \mathrm{IU} / \mathrm{L}$ is also predictive of the need for tube thoracostomy $[9,10]$.

\section{Case Report}

A 7-year-old boy, previously healthy, presented with vomiting, abdominal pain and high fever for 3 days. In ER he was toxic, febrile $\left(39.5^{\circ} \mathrm{C}\right)$ with moderate tachypnea, subcostal retractions and grunting. Diffuse crackles bilaterally. Blood tests: WBC: $13 \mathrm{k} / \mathrm{ul}$ (Normal range: 5 - $15 \mathrm{k} / \mathrm{ul}$ ); Neutrophils: $11.7 \mathrm{k} / \mathrm{ul}$ (Normal range: 1.5 - 6 k/ul); Lymphocytes: 0.8 k/ul (Normal range: 5 - 9 k/ul); Hemoglobin: 14 g/dl (Normal range: 10.5 - 14 g/dl); Platelets: 297 k/ ul (Normal range: 150 - 400 k/ul); Erythrocyte Sedimentation Rate (ESR): $81 \mathrm{~mm}$ (Normal range: < $15 \mathrm{~mm} / \mathrm{l}$ ); respiratory syncytial virus, influenza A and B swabs were negative and C-reactive Protein (CRP): $398 \mathrm{mg} / \mathrm{l}$ (Normal range: < $5 \mathrm{mg} / \mathrm{l}$ ). Moreover, electrolytes, liver and kidney functions were within normal.

Chest $\mathrm{X}$ ray, ultrasound and computed tomography at that time showed consolidation on left side and mild pleural effusion (Figure 1). Intravenous antibiotics (Ceftriaxone and Linezolid) were started and oxygen was continued through face mask. Day 5 Chest ultrasound showed a marked increase in the pleural fluid on the left side; therefore, an intercostal tube was inserted, and $250 \mathrm{ml}$ of purulent fluid was drained from the left side. The intercostal tube was left inside for 7 days (Figure 2). Pleural fluid analysis, histology and C/S results: WBCs 19400 cells/ul, RBCs 1500 cells/ul. Cytology (puss cells, macrophages, neutrophils, mesothelial cells no malignant cells. Gram stain negative. AFB stain: negative. Culture: no bacterial growth detected.

The complete resolution was confirmed by ultrasound and chest x ray (Figure 3).

Day 14 follow up ultrasound showed an increase of pleural fluid on the right side therefore, chest tube was inserted and $175 \mathrm{ml}$ of

Citation: Gihad Alsaeed., et al. "Recurrent Empyema in a Healthy 7 Year Old Boy". Acta Scientific Paediatrics 3.7 (2020): 54-60. 


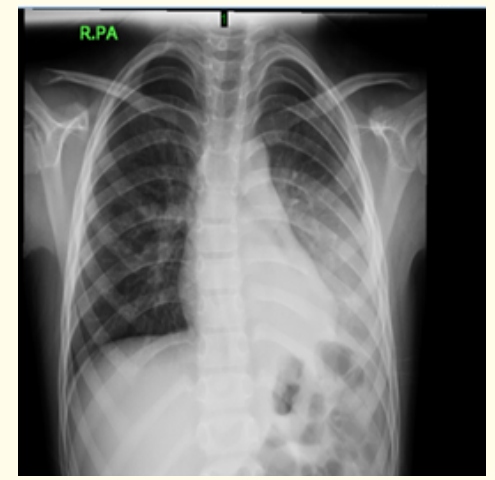

Figure 1: Left side lobar pneumonia with mild pleural effusion.

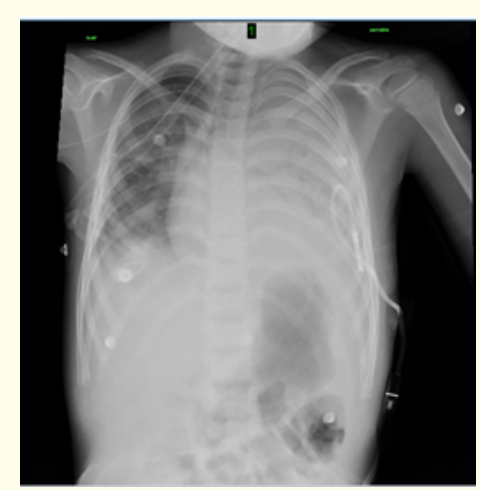

Figure 2: Left side huge pleural effusion and pig tail chest tube in situ.

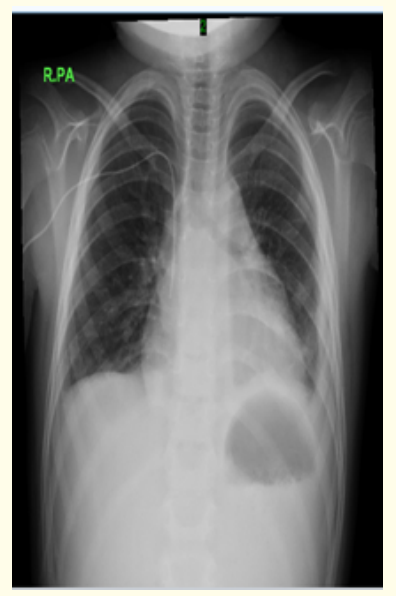

Figure 3: Resolution of pleural effusion.

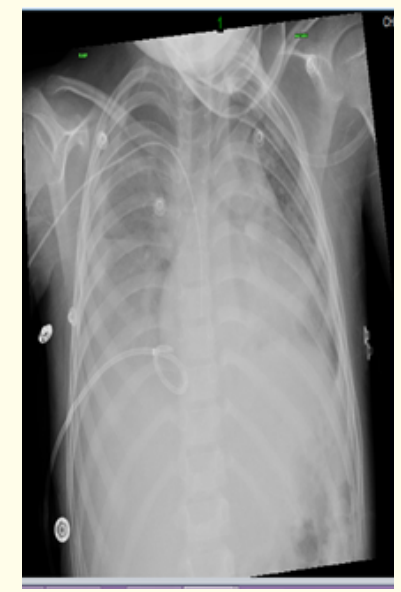

Figure 4: Right side pleural effusion with pig tail tube and central line in situ.

purulent fluid was drained from the right side (Figure 4).The intercostal tube was left inside for 5 days until the complete resolution was confirmed by ultrasound. Intravenous Linezolid and Ceftriaxone continued for total of 21 days. Then switched to oral Linezolid and Cefpodoxime for 10 days more. Having the results of all cultures as no growth including pleural fluid, blood, urine and throat cultures with normal inflammatory markers, the case considered recovered.

26 days after discharge he had high fever again, abdominal pain, vomiting and poor oral intake without cough. He was toxic boy, febrile $\left(39^{\circ} \mathrm{C}\right)$ with abdominal tenderness and bilaterally diffuse crackles. Chest imaging (X ray, ultrasound, echocardiography and computed tomography) showed that he had bilateral bronchopneumonia changes, bilateral empyema mainly on the left side and pericardial effusion (Figure 5). patient was admitted again, intravenous (Vancomycin and Meropenem), fluids and supplemental oxygen by mask were started.

Laboratory results: WBC: $16 \mathrm{k} / \mathrm{ul}$ (Normal range: 5 - $15 \mathrm{k} / \mathrm{ul}$ ); Neutrophils: $8.7 \mathrm{k} / \mathrm{ul}$ (Normal range: 1.5 - $6 \mathrm{k} / \mathrm{ul}$ ); Lymphocytes: $5.3 \mathrm{k} / \mathrm{ul}$ (Normal range: 5 - $9 \mathrm{k} / \mathrm{ul}$ ); Hemoglobin: $11.5 \mathrm{~g} / \mathrm{dl}$ (Normal range: 10.5 - 14 g/dl); Platelets: 224 k/ul (Normal range: 150 400 k/ul); Erythrocyte Sedimentation Rate (ESR): 42 mm (Normal range: $<15 \mathrm{~mm} / \mathrm{l}$ ); respiratory syncytial virus, influenza $\mathrm{A}$ and $\mathrm{B}$ swabs were negative, C-reactive Protein (CRP): $72 \mathrm{mg} / \mathrm{l}$ (Normal range: $<5 \mathrm{mg} / \mathrm{l}$ ). Moreover, electrolytes, liver and kidney functions were within normal. Pericardiosynthesis with needle aspiration of 

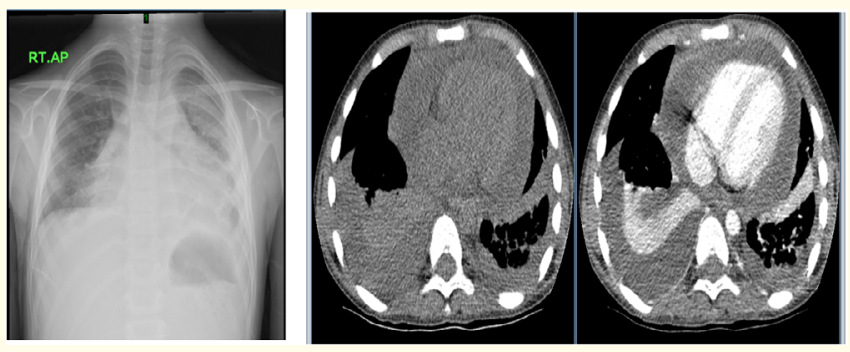

Figure 5: Chest $x$ ray and CT-scan: pericardial and pleural effusions and loculation formation.

$125 \mathrm{ml}$, Plus Pig tail chest tube was inserted and left for 6 days. 500 $\mathrm{ml}$ of purulent fluid was drained . Pleural fluid analysis: WBCs 9275 cells/ul, RBCs 980. Reactive mesothelial cells, macrophages, and acute inflammatory cells (neutrophilic exudate). No malignant cells. Negative culture and gram stain. Pericardial fluid: WBC 1500 cells/ul, RBCs 180 cells/ul. Inflammatory cells. No bacterial growth detected by culture.

Immunology screening including immunoglobulins IGA 1.68 g/l, IGE 305 g/l, IGM 0.87 g/l, IGG 7.65 g/l, immunoglobulin G subclasses $(1=5.9,2=1.59,3=0.46,4=0.112 \mathrm{~g} / \mathrm{l})$, tetanus $0.82 \mathrm{IU} /$ $\mathrm{ml}$ and diphtheria antibodies $1 \mathrm{IU} / \mathrm{ml}$ were satisfying.

Lymphocyte subsets were within normal as shown below:

- $\quad$ Lymphocytes, absolute count: $3885 / \mu \mathrm{l}$

- $\quad$ Ref. range: 1100 - 5900

- T-Lymphocytes (CD3+): 3188/ $\mu \mathrm{l}$

- $\quad$ Ref. rang (700 - 4200)

- $\quad$ T Lymphocytes (CD3+): 82+ \% lymph.

- $\quad$ Ref. range: (55 - 78)

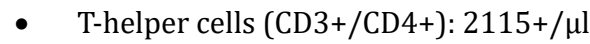

- Ref. range: (300 - 2000)

- T helper cells (CD3+/CD4+): 54+ \% lymph

- $\quad$ Ref. range: (27 - 53)

- $\quad$ T-suppressor cells (CD3+/CD8+): 945/ $\mu \mathrm{l}$

- $\quad$ Ref. range: (300 - 1800)

- T suppressor cells (CD3+/CD8+): 24\% lymph.
- $\quad$ Ref. range: $(19-34)$

- $\quad$ CD4+/CD8+ ratio: 2.2 (0.90 - 2.6)

- $\quad$ ABS Total T CD3 $3600 \mathrm{H} \mathrm{mm}^{3}(1,700$ - 1,900)

- $\quad$ Total T CD3 83\%

- $\quad$ ABS Helper T CD4 $2534 \mathrm{H} \mathrm{mm}^{3}(800-1,700)$

- $\quad$ Helper T CD4 58\%

- $\quad$ ABS Sup Cyto T CD8 $920 \mathrm{~mm}^{3}$ (700 - 1,000)

- $\quad$ Sup Cyto T CD8 21\%

- $\quad$ ABS Total B CD19 i $477 \mathrm{~mm}^{3}$ (400 - 800)

- $\quad$ Total B CD19 i 11\%

- $\quad$ ABS Total NK CD56 and $16254 \mathrm{~mm}^{3}$ (200 - 400)

- $\quad$ Total NK CD56 and $16.6 \%$

- $\quad$ CD4/CD8 Ratio 2.8 H (0.9 - 1.4)

- $\quad$ Total MHC Class II 13\%

- $\quad$ MHC Class II CD19DUAL Plus 11\%.

Antinuclear antibodies (7.83 U/ml), complement C3 (1.87 g/l) and C4 (0.34 g/l) were also normal. Blood film showed Neutrophilia with toxic granulation but there was no blast cells.

At day 28 of the second admission, the patient started to have fever again with abdominal pain and nausea. Re-evaluation showed deterioration in his laboratory values again with high C-reactive Protein (CRP): $144 \mathrm{mg} / \mathrm{l}$ (Normal range: $<5 \mathrm{mg} / \mathrm{l}$ ). Chest $\mathrm{x}$ ray and CT showed that the empyema recurred on the left side and pig tail tube inserted again for the $3^{\text {rd }}$ time on the left side (Figure 6). Within 3 days he improved dramatically and intravenous Vancomycin + Meropenem was continued for 6 weeks. Then discharged on oral Linezolid and Cefixime for 3 weeks more. The patient has been on regular follow up over the past 2 years . He is symptom free, with normal lung functions and chest $\mathrm{x}$ ray (Figure 7).

\section{Discussion}

Non-invasive investigation procedures to evaluate empyema are chest X ray, ultrasound, computed tomography of the chest. Ultrasonography is the preferred imaging [11]. The presence of septations and cellular debris and the lack of free movement with gravity suggest a complicated (fibrinopurulent) effusion. Ultrasonography is superior to CT because of better accuracy in detecting early loculations and septations. Computed tomography (CT) is not routinely 


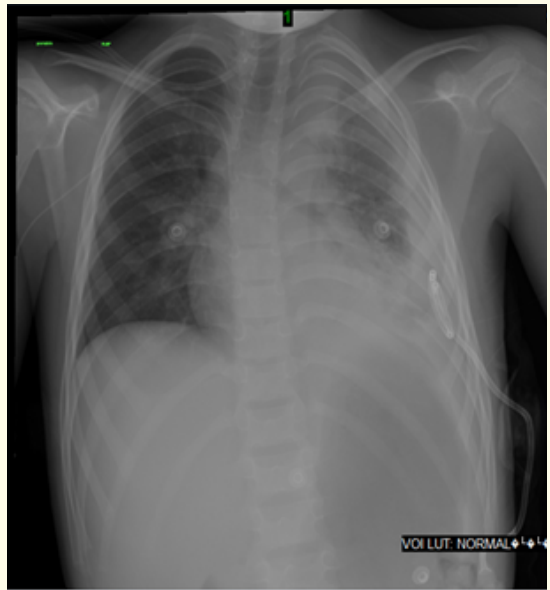

Figure 6: Recurrent pleural empyema with pig tail in situ.

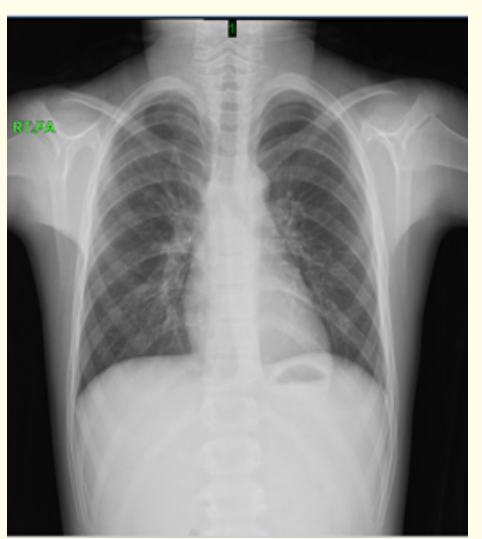

Figure 7: Normal chest $\mathrm{x}$ ray after 6 months.

necessary in the evaluation of children with parapneumonic effusion/empyema, and its use is generally limited to cases in which there is clinical suspicion for lung necrosis or abscess formation or to rule out other causes for effusion [12]. Drainage of pleural fluid is indicated for some moderate and most large pleural effusions. For patients with no respiratory compromise and with small to moderate pleural effusions, treatment with antibiotics but without pleural drainage is a reasonable alternative [2]. Pleural fluid findings in favor of pleural empyema rather than pleural effusion are : frank pus or bacteria in the pleural fluid, increased white blood cells, LDH, protein and fibrin. Pleural fluid must be sent for microbiologic analysis including Gram stain and bacterial culture [13]; these were reported to be positive in up to 49 percent of cases, with most studies reporting positive culture in less than 25 percent of cases [2]. However, like our patient, pleural fluid cultures are often sterile because of prior administration of antibiotics. If a broad-range PCR is not available, specific testing should be done for $S$. pneumoniae (pneumococcus) and S. pyogenes. The differential cell count can be helpful in determining an etiology; Mononuclear predominance suggests malignancy or tuberculosis [14]. Although some characteristics are associated with empyema $(\mathrm{pH}<7.0$, glucose $<40 \mathrm{mg} / \mathrm{dL}[2.2 \mathrm{mmol} / \mathrm{L}]$, lactate dehydrogenase $>1000$ international units), these measures do not help with decision-making in most cases $[9,13]$. Instead, the decision to drain a parapneumonic effusion can be made based on clinical criteria. Management of parapneumonic effusion depends on the stage of evolution of the parapneumonic process and what therapy has already been provided. In some cases, oral antibiotics may have already been initiated in the outpatient setting before the effusion became apparent. Inspite of oral or parenteral treatment, The effusion may become larger and/or compromise respiratory function thus it requires hospitalization, intravenous (IV) antibiotics, ultrasound assessment, and drainage of pleural effusion [15].

Treatment of empyema usually requires more aggressive therapy, including fibrinolytic therapy or surgical debridement/drainage of the pleural space $[14,15]$. Primary operative therapy was defined as children being treated initially with antibiotics and either VATS or thoracotomy, and primary nonoperative therapy was defined as children being treated initially with antibiotics and thoracentesis and/or tube thoracostomy [15].

The PubMed (National Library of Medicine) database for the period from 1981 to 2004. Reproducible search identified all publications dealing with treatment of empyema in the pediatric population ( $<18$ years of age) [5]. These aggregate results suggest that primary operative therapy is associated with a lower in-hospital mortality rate, re intervention rate, length of stay, time with tube thoracostomy and time of antibiotic therapy, compared with nonoperative treatment. The meta-analysis demonstrates a significantly reduced relative risk of failure among patients treated operatively [15]. Preferred empiric therapy is IV ceftriaxone or cefotaxime (where available), PLUS clindamycin or vancomycin. The duration of antibiotic therapy depends on the drainage and clinical response $[14,15]$. Common practice is to continue antibiotics for at least 10 days after resolution of fever; antibiotics may be changed from the IV to oral route when the child has been afebrile and without 
a chest drain for two to five days. Usually a total antibiotic course of two to four weeks is adequate [5]. However, certain pathogens, including CA-MRSA, may require a longer course of treatment.

Regardless of which treatment is used in empyema, the outcome for children is generally excellent. The aim of treatment is to resolve clinical symptoms and prevent further progression of empyema, sterilize the pleural cavity, reduce fever, shorten hospital stay and re-expand the lung with return to normal function. This often requires fluid drainage. Multi-disciplinary team should be involved in the management of this case (pediatrician, intervention radiologist, pulmonologist, pediatric cardiologist, immunologist and pediatric surgeon). Risk factors for empyema: failure to thrive, a history of chronic illness, congenital or chromosomal abnormality, anatomic or functional asplenia, immuno-compromise, previous invasive pneumococcal disease, prematurity and parental smoking history. Recurrent empyema in children is usually associated with recurrent pneumonia, chronic pyogenic lung disease, chest surgeries, immunodeficiency, or chest wall infections. In some cases recurrent empyema cannot be explained even by extensive study $[1,5,16]$.

\section{Conclusion}

Almost one third of hospitalized pneumonias in children are complicated by parapneumonic effusions which once infected turns into empyema .which pleural effusion will develop to empyema in previously healthy children seems unpredictable. Primary operative therapy has lower in-hospital mortality rate, re-intervention rate, length of stay, time with tube thoracostomy, and time of antibiotic therapy, compared with nonoperative treatment. 8.9\% of empyema thoracic recur after its apparent recovery. Recurrent empyema usually needs open or video assisted chest surgery with long period of parenteral antibiotics to recover. Such cases often associate chronic pyogenic lung disease, chest wall osteomyelitis or immunodeficiency. Most cases of empyema in children recover with normal lung functions and chest $x$ ray. Recurrent empyema in healthy children seems to have good prognosis even with nonoperative option. Further studies about non operative treatment of recurrent empyema in children are needed.

\section{Bibliography}

1. Byington CL., et al. "An epidemiological investigation of a sustained high rate of pediatric parapneumonic empyema: risk factors and microbiological associations". Clinical Infectious Diseases 34 (2002): 434.
2. BP Kuti and OA Oyelami. "Risk factors for parapneumonic effusions among children admitted with community-acquired pneumonia at a tertiary hospital in south-west Nigeria". The African Journal of Respiratory Medicine 10.1 (2014).

3. AF Saleem., et al. "Empyema Thoracis in Children: Clinical Presentation, Management and Complications". Journal of College of Physicians and Surgeons Pakistan 24.8 (2014): 573-576.

4. Narendra and Daizy. "Empyema Thoracis In Children: A Clinical Study". Journal of Evolution of Medical and Dental Sciences 5 (2016).

5. IM Balfour-Lynn., et al. "BTS guidelines for the management of pleural infection in children". Thorax 60.11 (2005): i1-i21.

6. KR Shen., et al. "The American Association for Thoracic Surgery consensus guidelines for the management of empyema". The Journal of Thoracic and Cardiovascular Surgery 153.6 (2017): e129-e146.

7. Wiese AD., et al. "Changes in empyema among U.S. children in the pneumococcal conjugate vaccine era". Vaccine 34 (2016): 6243.

8. SK Angurana., et al. "Pediatric empyema thoracis: What has changed over a decade?" Journal of Tropical Pediatrics's 65.3 (2018): 231-239.

9. JE Heffner., et al. "Pleural fluid chemical analysis in parapneumonic effusions. A meta-analysis". American Journal of Respiratory and Critical Care Medicine 151.6 (1995): 1700-1708.

10. RW Light., et al. "Parapneumonic effusions". The American Journal of Medicine 69.4 (1980): 507-512.

11. Islam S., et al. "The diagnosis and management of empyema in children: a comprehensive review from the APSA Outcomes and Clinical Trials Committee". Journal of Pediatric Surgery 47 (2012): 2101.

12. Yang PC., et al. "Value of sonography in determining the nature of pleural effusion: analysis of 320 cases". AJR. American Journal of Roentgenology 159 (1992): 29.

13. Balfour-Lynn IM., et al. "BTS guidelines for the management of pleural infection in children". Thorax 60.1 (2005): i1. 
14. AT Society. "Management of nontuberculous empyema". The American Review of Respiratory Disease Returns 85 (1962): 935-936.

15. RE Strachan and A Jaffé. "Recommendations for managing paediatric empyema thoracis". Medical Journal of Australia 195.2 (2011): 95.

16. PP España., et al. "A prediction rule to identify allocation of inpatient care in community-acquired pneumonia". European Respiratory Journal 21.4 (2003): 695-701.

\section{Assets from publication with us}

- Prompt Acknowledgement after receiving the article

- Thorough Double blinded peer review

- Rapid Publication

- Issue of Publication Certificate

- High visibility of your Published work

Website: www.actascientific.com/

Submit Article: www.actascientific.com/submission.php

Email us: editor@actascientific.com

Contact us: +919182824667 